\title{
Um problema de navegação de Zermelo: Métrica de Funk
}

\author{
A Zermelo navigation problem: Funk Metric
}

Newton Mayer Solórzano Chávez

Universidade Federal da Integração Latino-Americana (UNILA), Instituto Latino-Americano de Ciências da Vida e da Natureza (ILACVN), Foz do Iguaçu, PR, Brasil

https: //orcid.org/0000-0001-5492-2068, nmayer159@gmail.com Víctor Arturo Martínez León

Universidade Federal da Integração Latino-Americana (UNILA), Instituto Latino-Americano de Ciências da Vida e da Natureza (ILACVN), Foz do Iguaçu, PR, Brasil https://orcid.org/0000-0002-2082-6665, victor.leon@unila.edu.br Luz Gisselle Quevedo Sosa

Universidade Federal da Integração Latino-Americana (UNILA), Instituto Latino-Americano de Tecnologia, Infraestrutura e Território (ILATIT), Foz do Iguaçu, PR, Brasil https://orcid.org/0000-0001-5997-8204, luz.sosa@aluno.unila.edu.br Junior Rodrigues Moyses

Universidade Federal da Integração Latino-Americana(UNILA), Instituto Latino-Americano de Ciências da Vida e da Natureza (ILACVN), Foz do Iguaçu, PR, Brasil https://orcid.org/0000-0002-6754-7145, jr.moyses.2016@aluno.unila.edu.br

\section{Informações do Artigo}

\section{Como citar este artigo}

CHÁVEZ, Newton Mayer Solórzano; LEÓN, Víctor Arturo Martínez; SOSA, Luz Gisselle Quevedo; MOYSES, Junior Rodrigues. Um problema de navegação de Zermelo: Métrica de Funk. REMAT: Revista Eletrônica da Matemática, Bento Gonçalves, RS, v. 7, n. 1, p. e3010, 29 mar. 2021. DOI: https:// doi.org/10.35819/remat2021v7i1id4574

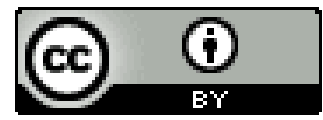

Histórico do Artigo

Submissão: 10 de dezembro de 2020.

Aceite: 10 de setembro de 2020.

\section{Resumo}

O artigo aborda um modelo específico de Geometria NãoEuclidiana, cujo disco aberto unitário centrado na origem do plano cartesiano é dotado de uma métrica de Randers, que modela o Problema da navegação de Zermelo. Com isso, é gerada a "Geometria de Funk sobre o disco unitário", para qual a distância não é simétrica. Nesse sentido, o estudo apresenta as expressões para distância de ponto a ponto - de ponto a uma linha reta, e de uma linha reta a um ponto; e caracteriza as circunferências nesse tipo de geometria. Exemplos explícitos são incluídos.

\section{Palavras-chave}

Métrica de Finsler

Métrica de Funk

Problema de Navegação 


\section{Keywords}

Finsler Metric

Funk Metric

Navigation Problem

\begin{abstract}
The article approaches a specific model of Non-Euclidean Geometry which unitary open disk centered at the origin of the cartesian plane is endowed with a Randers metric, which models the Zermelo's navigation problem. As a result, the "Funk Geometry on the unit disk" is engendered, for which the distance is nonsymmetric. In this respect, the study presents the expressions for distance from point to point - from point to straight line, and from a straight line to point; and characterizes the circumferences in this type of geometry. Explicit examples are included.
\end{abstract}

\section{Introdução}

As métricas de Randers são um caso particular das métricas de Finsler (ver Shen (2001)), que, por sua vez, são uma generalização das métricas Riemannianas. Os interessados, que se introduzem no mundo das métricas de Finsler, podem se sentir frustrados pelas abstrações e notações, sentindo falta de exemplos bem particulares. Com este trabalho, objetiva-se introduzir, de maneira amical ao leitor, um tipo específico de métrica de Randers sobre o plano de tal forma que os prérequisitos sejam mínimos. Deste modo, um estudante de graduação, que tenha estudado cálculo diferencial e geometria analítica, conseguirá acompanhar e entender os resultados do presente estudo.

Em 1931, Ernst Friedrich Ferdinand Zermelho ${ }^{1}$, propôs o Problema da Navegação de Zermelo, que consistia em um clássico problema de controle ótimo. Estes lidam com um barco navegando em um corpo de água, partindo de um ponto $A$ até um ponto de destino $B$. O barco é capaz de alcançar uma certa velocidade máxima, e procura-se obter o melhor controle possível para alcançar $B$ no menor tempo possível.

Descartando-se forças externas como a correnteza e o vento, o controle ótimo é seguir um segmento de reta de $A$ a $B$. Considerando correnteza e vento, o caminho mais curto de $A$ a $B$ não sempre é uma linha reta.

Este artigo aborda o caso particular de navegação, cujo modelo base desse estudo é apresentado no Exemplo 3.1. Por conseguinte, esse modelo é um caso particular das denominadas métricas de Funk definidas sobre um subconjunto conexo de $\mathbb{R}^{n}$. É possível mostrar (ver Exemplo

\footnotetext{
${ }^{1}$ E. Zermelho (Berlim, 27 de julho de 1871 - Friburgo, 21 de maio de 1953) foi um matemático e filósofo alemão, cujo trabalho teve influência direta nos Fundamentos da Matemática.
} 
9.2.1 em Shen (2001)) que os caminhos mais curtos na métrica de Funk são sempre linhas retas. Algumas questões sobre o modelo adotado surgem naturalmente, como, por exemplo, qual é o tempo mínimo requerido para chegar de uma ilha a outra? Ou dado uma quantidade fixa de combustível, até onde podemos chegar? Ou também qual a melhor rota para chegar numa praia e vice-versa?. Para essas perguntas, as respostas podem ser encontradas no estudo de distância de ponto a ponto, circunferências e distância de ponto a reta e vice-versa.

\section{Preliminares}

Nesta seção, daremos algumas definições e resultados que nos serão de utilidade para o desenvolvimento do trabalho. O plano $\mathbb{R}^{2}$ será considerado como o conjunto de pares ordenados $\left(x_{1}, x_{2}\right)$, onde $x_{1}$ e $x_{2}$ são números reais.

Definição 2.1 (Produto interno e norma euclidiana em $\left.\mathbb{R}^{2}\right)$. Sejam $x=\left(x_{1}, x_{2}\right)$ e $\tilde{x}=\left(\tilde{x}_{1}, \tilde{x}_{2}\right)$ pontos de $\mathbb{R}^{2}$, o produto interno entre $x$ e $\tilde{x}$, denotado por $\langle x, \tilde{x}\rangle$, é o número definido por:

$$
\langle x, \tilde{x}\rangle=x_{1} \tilde{x}_{1}+x_{2} \tilde{x}_{2}
$$

A norma de $x$, denotada por $\|x\|$ é definida por:

$$
\|x\|=\sqrt{\langle x, x\rangle} .
$$

Definição 2.2 (Campo vetorial). Um campo vetorial em um subconjunto aberto $\Omega$ de $\mathbb{R}^{2}$ é uma aplicação $V: \Omega \subset \mathbb{R}^{2} \rightarrow \mathbb{R}^{2}$ que, associa a cada ponto $x \in \Omega$ um vetor $V(x)$ de $\mathbb{R}^{2}$.

Para não carregar notação, o campo vetorial $V(x)$ será denotado por $V_{x}$. Da mesma maneira, é conveniente denotar por $\Omega$ um subconjunto aberto de $\mathbb{R}^{2}$.

Definição 2.3 (Curva diferenciável parametrizada regular em $\Omega \subset \mathbb{R}^{2}$ ). Uma curva parametrizada em $\Omega \subset \mathbb{R}^{2}$ é uma aplicação $\alpha: I \rightarrow \Omega$ definida em um intervalo $I$ de $\mathbb{R}$. Uma curva parametrizada $\alpha$ é chamada diferenciável se $\alpha$ for de classe $C^{k}$ para algum $k=1,2, \ldots$, isto é, cada coordenada de $\alpha$ é de classe $C^{k}$ (ver p. 104 em Lima (2014)). Dizemos que uma curva parametrizada é suave se é de classe $C^{k}$ para todo $k=1,2, \ldots$. Uma curva parametrizada diferenciável $\alpha$ é chamada regular em $I$ se $\alpha^{\prime}(t) \neq(0,0)$ para todo $t \in I$. Uma curva parametrizada $\alpha$ é dita regular por partes se existem $t_{1}, t_{2}, \ldots, t_{n} \in I$ tal que $\left.\alpha\right|_{] t_{i}, t_{i+1}[}$ é regular em $] t_{i}, t_{i+1}[$, para cada $i=1, \ldots, n-1$.

Para a seguinte definição, consideremos que a curva possua primeira derivada e seja continua, isto é, a curva é de classe $C^{1}$. 
Definição 2.4 (Comprimento de arco em $\mathbb{R}^{2}$ ). Seja $\left.\alpha:\right] a, b\left[\rightarrow \mathbb{R}^{2}\right.$ uma curva parametrizada regular de classe $C^{1}$. A função comprimento de arco de $\alpha$ é definida por:

$$
s(t)=\int_{t_{0}}^{t}\left\|\alpha^{\prime}(\tau)\right\| \mathrm{d} \tau
$$

onde $\left.t_{0} \in\right] a, b[$ é dado.

Neste caso, observe que, pelo Teorema Fundamental do Cálculo (ver Teorema 1 na p. 137 em Lima (2014)), tem-se:

$$
s^{\prime}(t)=\left\|\alpha^{\prime}(t)\right\| \neq 0 .
$$

Definições formais da métricas Riemannianas são encontradas em vários livros avançados de geometria Riemanniana (veja Carmo (2011)) e geometria diferencial (ver Carmo (2012)). Para nosso propósito, restringimos tais definições sobre $\Omega \subset \mathbb{R}^{2}$ de tal forma que seja suficiente para 0 desenvolvimento do presente trabalho.

Definição 2.5 (Métrica Riemanniana). Para cada $i=1,2$ e $j=1,2$, sejam $a_{i j}: \Omega \subset \mathbb{R}^{2} \rightarrow \mathbb{R}$ funções diferenciáveis tais que a matriz $\left[a_{i j}(x)\right]_{2 \times 2}$ seja simétrica e positiva definida para todo $x \in \Omega$. Dado $x \in \Omega$ e qualquer vetor $y_{x}=\left(y_{1}, y_{2}\right) \in \mathbb{R}^{2}$, a quantidade

$$
\alpha\left(x, y_{x}\right)=\sqrt{a_{11}(x) y_{1}^{2}+2 a_{12}(x) y_{1} y_{2}+a_{22}(x) y_{2}^{2}}
$$

é chamada métrica Riemanniana sobre $\Omega \subset \mathbb{R}^{2}$.

Note que $\alpha$ generaliza a definição de norma euclidiana, pois basta considerar $a_{11}=a_{22}=1$ e $a_{12}=a_{21}=0$.

A seguinte definição pode ser interpretada como uma perturbação de uma métrica Riemanniana $\alpha$ por uma aplicação (linear em $y$ ) $\beta(x, y)=b_{1}(x) y_{1}+b_{2}(x) y_{2}$.

Definição 2.6 (Métrica de Randers). Considere $\alpha$ uma métrica Riemanniana sobre $\Omega$ e $\beta(x, y)=$ $b_{1}(x) y_{1}+b_{2}(x) y_{2}$, com $b_{1}, b_{2}: \Omega \rightarrow \mathbb{R}$ funções diferenciáveis tais que:

$$
\sqrt{a^{11}(x)\left[b_{1}(x)\right]^{2}+\left[a^{12}(x)+a^{21}(x)\right] b_{1}(x) b_{2}(x)+a^{22}(x)\left[b_{2}(x)\right]^{2}}<1,
$$

onde $\left[a^{i j}(x)\right]=\left[a_{i j}(x)\right]^{-1}$, para todo $x \in \Omega$. Uma métrica de Randers sobre $\Omega \subset \mathbb{R}^{2}$, é uma função $F: \Omega \times \mathbb{R}^{2} \rightarrow \mathbb{R}$ definida por:

$$
F\left(x, y_{x}\right)=\alpha\left(x, y_{x}\right)+\beta\left(x, y_{x}\right)
$$


Maiores detalhes sobre as métricas de Randers encontram-se no Capítulo 2 de Cheng e Shen (2012).

Definição 2.7 (Comprimento de arco do tipo Randers). Seja $c:[a, b] \rightarrow \Omega \subset \mathbb{R}^{2}$ uma curva regular por partes. O comprimento de arco (tipo Randers) de $c$ é definido por:

$$
\mathcal{L}_{F}(c):=\int_{a}^{b} F\left(c(t), c^{\prime}(t)\right) d t
$$

Para quaisquer pontos $p, q \in \Omega \subset \mathbb{R}^{2}$, definimos a distância de $p$ até $q$ induzida por $F$, como:

$$
d_{F}(p, q):=\inf _{c} \mathcal{L}_{F}(c)
$$

onde o ínfimo é tomado sobre o conjunto de todas as curvas regulares por partes $c$ tais que $c(a)=p$ $\mathrm{e} c(b)=q$.

Observação 2.8. (ver p. 14 em Cheng e Shen (2012)) A quantidade $d_{F}=d_{F}(p, q)$ é uma função não negativa sobre $\Omega \times \Omega \subset \mathbb{R}^{2} \times \mathbb{R}^{2}$, e satisfaz:

- $d_{F}(p, q) \geq 0$. A igualdade é satisfeita se, e somente se, $p=q$.

- $d_{F}(p, q) \leq d_{F}(p, r)+d_{F}(r, q)$ para quaisquer $p, q, r \in \Omega \subset \mathbb{R}^{2}$.

Isso justifica a designação do nome de distância induzida por $F$ para $d_{F}$.

\section{Problema de Navegação de Zermelo}

É conveniente mencionar que qualquer problema de navegação de Zermelo em $\Omega \subset \mathbb{R}^{2}$ (inclusive em domínios maiores como variedades diferenciáveis) induz uma métrica de Randers em $\Omega$ (ou em domínios maiores). E mais do que isso, qualquer métrica de Randers provém de um problema de navegação (ver Capítulo 2 em Cheng e Shen (2012)).

Nesta seção, estudaremos as Métrica de Randers que são obtidas a partir do problema de navegação de Zermelo modelado em um subconjunto aberto $\Omega$ de $\mathbb{R}^{2}$.

Suponha um objeto (um barco, por exemplo) na posição $x \in \Omega$ tenha o vetor velocidade interno $U_{x}$ (induzida pela força do motor por exemplo), de comprimento constante, $\left\|U_{x}\right\|=1$. Sem vetor velocidade externo (induzida pela força do vento por exemplo) os segmentos de reta são os caminhos mais curtos. Note que, nesse caso, o comprimento de um segmento de reta é exatamente 
o tempo de percurso do objeto, de fato, se $\alpha:\left[0, t_{0}\right] \rightarrow \mathbb{R}^{2}$ é o vetor posição do objeto tal que $\alpha^{\prime}(t)=U_{\alpha(t)}$ (vetor velocidade unitária), então:

$$
\int_{0}^{t}\left\|\alpha^{\prime}(\tau)\right\| d \tau=\int_{0}^{t} 1 d \tau=t
$$

Agora, suponhamos que existe um vetor velocidade externo $W_{x}$ de comprimento $\left\|W_{x}\right\|<1$. Esta condição é para assegurar que o objeto consiga se movimentar em todas as direções. A combinação dos dois vetores velocidades no objeto (ver Figura 1):

$$
T_{x}=U_{x}+W_{x}
$$

nos dá a direção e velocidade (ou força total induzida) do objeto no ponto $x \in \Omega$.

Figura 1 - Vetor velocidade resultante $T_{x}$.

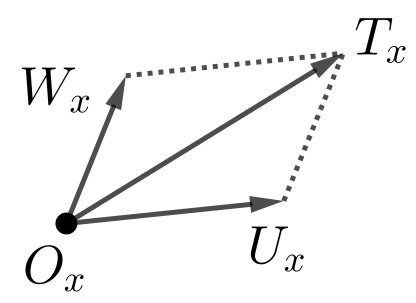

Fonte: Elaboração dos autores (2020).

Uma vez escolhido o vetor velocidade interno $U_{x}$ com $\left\|U_{x}\right\|=1$, temos:

$$
\left\|T_{x}-W_{x}\right\|=\left\|U_{x}\right\|=1
$$

Por outro lado, como se ilustra na Figura 2, é possível mostrar que, para qualquer vetor $y_{x} \in \mathbb{R}^{2}$, existe uma única solução $F=F\left(x, y_{x}\right)>0$ para a seguinte equação:

$$
\left\|\frac{y_{x}}{F\left(x, y_{x}\right)}-W_{x}\right\|=1 \text {. }
$$


Figura 2 - Existência de $F>0$.

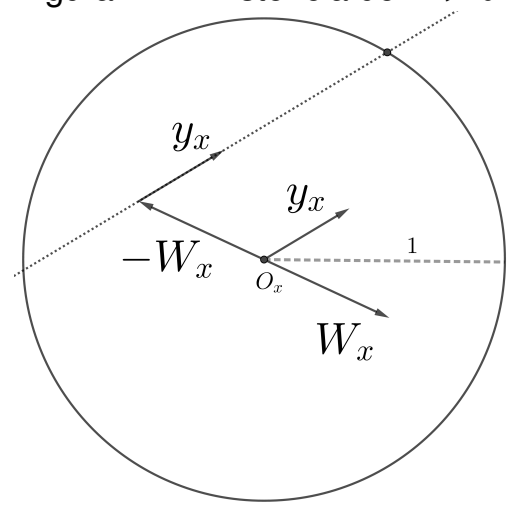

Fonte: Elaboração dos autores (2020).

Note que (ver Figura 3), para qualquer escalar $\lambda>0$, temos:

$$
1=\left\|\frac{\lambda y_{x}}{\lambda F\left(x, y_{x}\right)}-W_{x}\right\|=\left\|\frac{\lambda y_{x}}{F\left(x, \lambda y_{x}\right)}-W_{x}\right\| .
$$

Pela unicidade de $F$ obtemos:

$$
\lambda F\left(x, y_{x}\right)=F\left(x, \lambda y_{x}\right)
$$

Figura $3-F$ é homogênea em $y_{x}$.

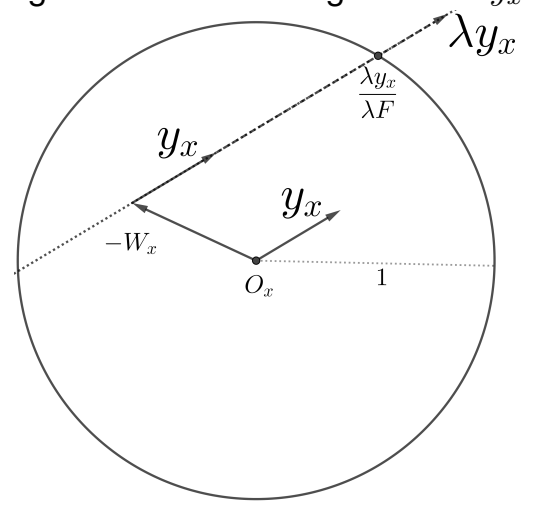

Fonte: Elaboração dos autores (2020).

Concluímos que $F=F\left(x, y_{x}\right)$ é uma função homogênea positiva de grau 1 em $y_{x} \in \mathbb{R}^{2}$ em cada ponto $x \in \Omega \subset \mathbb{R}^{2}$.

Comparando (3) e (4), obtemos:

$$
F\left(x, T_{x}\right)=1 .
$$


Se $c:\left[0, t_{0}\right] \rightarrow \Omega$ é uma curva suave com $c^{\prime}(t)=T_{c(t)}$, então o comprimento de arco $\mathcal{L}_{F}(c)$ de $c$ é igual ao tempo de viagem do objeto ao longo de $c$. De fato:

$$
\mathcal{L}_{F}(c)=\int_{0}^{t_{0}} F\left(c(\tau), T_{c(\tau)}\right) d \tau=\int_{0}^{t_{0}} 1 d \tau=t_{0}
$$

Assim, diante de uma força externa $W_{x}$, a procura de caminhos mais curtos não será mais na métrica euclidiana, e sim na métrica $F$.

Obtemos, na continuação, uma expressão para a função $F=F\left(x, y_{x}\right)$. Para simplificar notações, de agora em diante, retiramos o subíndice $x$ de $y_{x}$ e $W_{x}$. Elevando ao quadrado ambos os lados da equação (4) e abrindo a norma ao quadrado, obtemos:

$$
\frac{\|y\|^{2}}{F^{2}}-2 \frac{\langle y, W\rangle}{F}+\|W\|^{2}=1
$$

Agora, multiplicando $F^{2}$ os dois lados da igualdade acima temos:

$$
\left(1-\|W\|^{2}\right) F^{2}+2\langle y, W\rangle F-\|y\|^{2}=0 .
$$

Essa última igualdade é uma equação de segundo grau, cujas raízes são dadas por:

$$
F=-\frac{\langle W, y\rangle}{1-\|W\|^{2}} \pm \frac{\sqrt{\langle W, y\rangle^{2}+\|y\|^{2}\left(1-\|W\|^{2}\right)}}{1-\|W\|^{2}} .
$$

Agora, como:

$$
\sqrt{\langle W, y\rangle^{2}+\|y\|^{2}\left(1-\|W\|^{2}\right)} \geq|\langle W, y\rangle| \geq\langle W, y\rangle
$$

A igualdade é válida se, e somente se, $y=0$. Por tanto, sempre haverá uma raiz positiva e outra não positiva, desde que $F>0$ para todo $y \neq 0$ obtemos:

$$
F=\frac{\sqrt{\langle W, y\rangle^{2}+\|y\|^{2}\left(1-\|W\|^{2}\right)}}{1-\|W\|^{2}}-\frac{\langle W, y\rangle}{1-\|W\|^{2}} .
$$

Note que a expressão (5) é uma métrica de Randers $F=\alpha+\beta$ (ver (2)) com

$$
\alpha(x, y)=\frac{\sqrt{\langle W, y\rangle^{2}+\|y\|^{2}\left(1-\|W\|^{2}\right)}}{1-\|W\|^{2}} \quad \text { e } \quad \beta(x, y)=-\frac{\langle W, y\rangle}{1-\|W\|^{2}} .
$$

Em ocasiones, é conveniente reescrever (5) da seguinte forma:

$$
F=\frac{\sqrt{\lambda h^{2}+W_{o}^{2}}}{\lambda}-\frac{W_{o}}{\lambda},
$$


onde

$$
W_{o}=\langle W, y\rangle, \quad \lambda=1-\|W\|^{2} \text { e } \quad h=\|y\|
$$

O seguinte exemplo é um caso particular das métricas de Funk, denominada métrica de Funk sobre o disco unitário $\mathbb{B}^{2}=\left\{x \in \mathbb{R}^{2} ;\|x\|<1\right\}$. Inicialmente, as métricas de Funk são definidas sobre domínios conexos em $\mathbb{R}^{n}$. A seção 4 está baseada neste exemplo. Portanto, pedimos muita atenção.

Exemplo 3.1 (Métrica de Funk sobre $\mathbb{B}^{2}$ ). Consideremos $\Omega=\mathbb{B}^{2}, y=\left(y_{1}, y_{2}\right) \in \mathbb{R}^{2}$ e $W=-x=$ $\left(-x_{1},-x_{2}\right)$ com $\|W\|<1$.

De (7), obtemos os parâmetros $\lambda, W_{0}$ e $h$ :

$$
\begin{aligned}
& \lambda=1-x_{1}^{2}-x_{2}^{2} \\
& W_{o}=\langle W, y\rangle=\left\langle\left(-x_{1},-x_{2}\right),\left(y_{1}, y_{2}\right)\right\rangle=-x_{1} y_{1}-x_{2} y_{2} \\
& h^{2}=y_{1}^{2}+y_{2}^{2} .
\end{aligned}
$$

Substituindo (8) na equação (6), temos:

$$
F=\frac{\sqrt{\left(1-x_{1}^{2}-x_{2}^{2}\right)\left(y_{1}^{2}+y_{2}^{2}\right)+\left(-x_{1} y_{1}-x_{2} y_{2}\right)^{2}}}{1-x_{1}^{2}-x_{2}^{2}}+\frac{x_{1} y_{1}+x_{2} y_{2}}{1-x_{1}^{2}-x_{2}^{2}} .
$$

A igualdade (9) pode ser reescrita na forma:

$$
F=\sqrt{a_{11}(x) y_{1}^{2}+2 a_{12}(x) y_{1} y_{2}+a_{22}(x) y_{2}^{2}}+b_{1}(x) y_{1}+b_{2}(x) y_{2},
$$

onde

$$
b_{1}=\frac{x_{1}}{1-x_{1}^{2}-x_{2}^{2}}, \quad b_{2}=\frac{x_{2}}{1-x_{1}^{2}-x_{2}^{2}}
$$

e

$$
\left[a_{i j}\right]=\frac{1}{\left(1-x_{1}^{2}-x_{2}^{2}\right)^{2}}\left(\begin{array}{cc}
1-x_{2}^{2} & x_{1} x_{2} \\
x_{1} x_{2} & 1-x_{1}^{2}
\end{array}\right)
$$

cuja inversa é dada por:

$$
\left[a^{i j}\right]=\left(1-x_{1}^{2}-x_{2}^{2}\right)\left(\begin{array}{cc}
1-x_{1}^{2} & -x_{1} x_{2} \\
-x_{1} x_{2} & 1-x_{2}^{2}
\end{array}\right)
$$

Convidamos os leitores a obter outros exemplos de métricas de Randers, considerando diversos $W$. 


\section{Distância induzida pela métrica de Funk}

A métrica de Funk (veja Funk (1929)), obtida no Exemplo 3.1, denominada métrica de Funk sobre $\mathbb{B}^{2}$, é um tipo especial das métricas de Randers. Algumas propriedades foram estudadas em Shen (2001) para casos mais gerais. Sabe-se que os caminhos mais curtos (shortest path) nesta métrica são linhas de reta (ver Exemplo 9.2.1 em Shen (2001)), isto é, o caminho ótimo é sempre uma linha reta. Esta propriedade torna tratável esta métrica em relação ao cálculo de distâncias.

Maiores detalhes sobre geodésicas e a relação com caminhos mais curtos podem ser encontradas na Seção 3.2 de Chern e Shen (2005) e Seção 2.3 de Cheng e Shen (2012).

De agora em adiante, trataremos a métrica de Funk sobre $\mathbb{B}^{2}$ (obtida no Exemplo 3.1).

Sejam $P, Q \in \mathbb{B}^{2} \subset \mathbb{R}^{2}$ pontos distintos. Considere a curva paramétrica $\alpha:[0,1] \rightarrow \mathbb{B}^{2}$ definida por:

$$
\alpha(t)=t Q+(1-t) P
$$

que une os pontos $P$ e $Q$. Note que $\alpha(0)=P$ e $\alpha(1)=Q$.

Derivando 10 em relação a $t$ :

$$
\alpha^{\prime}(t)=Q-P
$$

A distância de "Funk" de $P$ até $Q$, denotada por $d(P, Q)$, é dada por:

$$
\begin{aligned}
d(P, Q) & =\mathcal{L}_{F}(\alpha) \\
& =\int_{0}^{1} F\left(\alpha(t), \alpha^{\prime}(t)\right) d t
\end{aligned}
$$

onde $F$ é dada por (9). Substituindo 10 e 11 em (12), temos:

$$
d(P, Q)=d(P, Q)_{1}+d(P, Q)_{2},
$$

onde

$$
d(P, Q)_{1}=\int_{0}^{1} \frac{\sqrt{\|Q-P\|^{2}\left(1-\|P\|^{2}\right)+\langle P, Q-P\rangle^{2}}}{\left(1-\|P\|^{2}\right)-2\langle P, Q-P\rangle t-\|Q-P\|^{2} t^{2}} d t
$$

e

$$
d(P, Q)_{2}=\int_{0}^{1} \frac{\|Q-P\|^{2} t+\langle P, Q-P\rangle}{\left(1-\|P\|^{2}\right)-2\langle P, Q-P\rangle t-\|Q-P\|^{2} t^{2}} d t
$$

Definimos $k$, da seguinte forma, para simplificar notações:

$$
k=\|Q-P\|^{2}\left(1-\|P\|^{2}\right)+\langle P, Q-P\rangle^{2} .
$$


Note:

$\left(1-\|P\|^{2}\right)-2\langle P, Q-P\rangle t-\|Q-P\|^{2} t^{2}=-\|Q-P\|^{2}\left[t^{2}+2 \frac{\langle P, Q-P\rangle}{\|Q-P\|^{2}}-\frac{1-\|P\|^{2}}{\|Q-P\|^{2}}\right]$.

Agora, fatorizando o lado direito da igualdade acima, obtemos:

$$
\left(1-\|P\|^{2}\right)-2\langle P, Q-P\rangle t-\|Q-P\|^{2} t^{2}=-\|Q-P\|^{2}\left(t-t_{1}\right)\left(t-t_{2}\right),
$$

onde

$$
t_{1}=\frac{-\sqrt{\langle P, Q-P\rangle^{2}+\left(1-\|P\|^{2}\right)\|Q-P\|^{2}}-\langle P, Q-P\rangle}{\|Q-P\|^{2}}
$$

e

$$
t_{2}=\frac{\sqrt{\langle P, Q-P\rangle^{2}+\left(1-\|P\|^{2}\right)\|Q-P\|^{2}}-\langle P, Q-P\rangle}{\|Q-P\|^{2}} .
$$

Substituindo (16) e (17) em (14), temos:

$$
d(P, Q)_{1}=-\frac{\sqrt{k}}{\|Q-P\|^{2}} \int_{0}^{1} \frac{1}{\left(t-t_{1}\right)\left(t-t_{2}\right)} d t
$$

Dessa vez, utilizando o método de frações parciais, tem-se:

$$
d(P, Q)_{1}=-\frac{\sqrt{k}}{\|Q-P\|^{2}} \int_{0}^{1}\left[\frac{1}{\left(t_{1}-t_{2}\right)\left(t-t_{1}\right)}-\frac{1}{\left(t_{1}-t_{2}\right)\left(t-t_{2}\right)}\right] d t
$$

Logo, integrando, obtemos:

$$
\begin{aligned}
d(P, Q)_{1} & =-\frac{\sqrt{k}}{\|Q-P\|^{2}}\left[\frac{\ln \left|t-t_{1}\right|}{t_{1}-t_{2}}-\frac{\ln \left|t-t_{2}\right|}{t_{1}-t_{2}}\right]_{0}^{1} \\
& =-\frac{\sqrt{k}}{\|Q-P\|^{2}} \frac{1}{\left(t_{1}-t_{2}\right)}\left[\ln \left|\frac{t-t_{1}}{t-t_{2}}\right|\right]_{0}^{1} \\
& =-\frac{\sqrt{k}}{\|Q-P\|^{2}} \frac{1}{\left(t_{1}-t_{2}\right)} \ln \left|\frac{t_{2}\left(1-t_{1}\right)}{t_{1}\left(1-t_{2}\right)}\right|
\end{aligned}
$$

Observe que, substituindo (16) em (18) e (19), temos:

$$
\frac{1}{t_{1}-t_{2}}=-\frac{\|Q-P\|^{2}}{2 \sqrt{k}} \text {. }
$$

Assim, substituindo (21) em (20, temos:

$$
d(P, Q)_{1}=\frac{1}{2} \ln \left|\frac{t_{2}\left(1-t_{1}\right)}{t_{1}\left(1-t_{2}\right)}\right| .
$$


Por outro lado, nota-se

$$
\left[\left(1-\|P\|^{2}\right)-2\langle P, Q-P\rangle t-\|Q-P\|^{2} t^{2}\right]^{\prime}=-2\left[\|Q-P\|^{2} t+\langle P, Q-P\rangle\right] .
$$

Logo, substituindo (23) em (15), temos:

$$
d(P, Q)_{2}=-\frac{1}{2} \int_{0}^{1} \frac{\left[\left(1-\|P\|^{2}\right)-2\langle P, Q-P\rangle t-\|Q-P\|^{2} t^{2}\right]^{\prime}}{\left(1-\|P\|^{2}\right)-2\langle P, Q-P\rangle t-\|Q-P\|^{2} t^{2}} d t .
$$

Pelo Teorema Fundamental do Cálculo, obtemos:

$$
d(P, Q)_{2}=-\frac{1}{2}\left[\ln \left|\left(1-\|P\|^{2}\right)-2\langle P, Q-P\rangle t-\|Q-P\|^{2} t^{2}\right|\right]_{0}^{1} .
$$

Assim, substituindo (17) em 24, temos:

$$
d(P, Q)_{2}=-\frac{1}{2}\left[\ln \left|-\|Q-P\|^{2}\left(t-t_{1}\right)\left(t-t_{2}\right)\right|\right]_{0}^{1}
$$

ou, equivalentemente, obtemos:

$$
d(P, Q)_{2}=-\frac{1}{2} \ln \left|\frac{\left(1-t_{1}\right)\left(1-t_{2}\right)}{t_{1} t_{2}}\right| .
$$

Substituindo (22) e 25) em (13), temos:

$$
\begin{aligned}
d(P, Q) & =\frac{1}{2} \ln \left|\frac{t_{2}\left(1-t_{1}\right)}{t_{1}\left(1-t_{2}\right)}\right|-\frac{1}{2} \ln \left|\frac{\left(1-t_{1}\right)\left(1-t_{2}\right)}{t_{1} t_{2}}\right| \\
& =\frac{1}{2} \ln \left(\frac{t_{2}^{2}}{\left(1-t_{2}\right)^{2}}\right) \\
& =\ln \left|\frac{t_{2}}{t_{2}-1}\right| .
\end{aligned}
$$

Afirmação 4.1. $t_{2}>1$.

De fato, observe que, da definição de $t_{2}$ em (19), temos que $t_{2}>1$ se, e somente se,

$$
\sqrt{\langle P, Q-P\rangle^{2}+\left(1-\|P\|^{2}\right)\|Q-P\|^{2}}>\langle P, Q-P\rangle+\|Q-P\|^{2} .
$$

Agora, se apresentam duas situações:

- Se $\langle P, Q-P\rangle+\|Q-P\|^{2}<0$ então (27) claramente é verdadeira. 
- Se $\langle P, Q-P\rangle+\|Q-P\|^{2} \geq 0$, temos que 27] é verdadeira se, e somente se,

$$
\begin{gathered}
\langle P, Q-P\rangle^{2}+\left(1-\|P\|^{2}\right)\|Q-P\|^{2}>\langle P, Q-P\rangle^{2}+2\langle P, Q-P\rangle\|Q-P\|^{2}+\|Q-P\|^{4} \\
\Leftrightarrow\left(1-\|P\|^{2}\right)\|Q-P\|^{2}>2\langle P, Q-P\rangle\|Q-P\|^{2}+\|Q-P\|^{4}
\end{gathered}
$$

como $P \neq Q$, dividindo $\|Q-P\|^{2}$ ambos os lados da última desigualdade acima, temos:

$$
\begin{aligned}
1-\|P\|^{2}>2\langle P, Q-P\rangle+\|Q-P\|^{2} & \Leftrightarrow 1>\|P\|^{2}+2\langle P, Q-P\rangle+\|Q-P\|^{2} \\
& \Leftrightarrow 1>\|P+(Q-P)\|^{2} \\
& \Leftrightarrow 1>\|Q\|^{2},
\end{aligned}
$$

que também é verdadeira.

Portanto, em qualquer caso, 27] é verdadeira. Concluí-se a prova da afirmação.

Utilizando a Afirmação 4.1 em 26, temos que a distância de $P$ até $Q$ é dada por:

$$
d(P, Q)=\ln \left(\frac{t_{2}}{t_{2}-1}\right)
$$

Substituindo (19) em 28, obtemos:

$$
d(P, Q)=\ln \left(\frac{\sqrt{\langle P, Q-P\rangle^{2}+\left(1-\|P\|^{2}\right)\|Q-P\|^{2}}-\langle P, Q-P\rangle}{\sqrt{\langle P, Q-P\rangle^{2}+\left(1-\|P\|^{2}\right)\|Q-P\|^{2}}-\langle P, Q-P\rangle-\|Q-P\|^{2}}\right) .
$$

\section{Observação 4.2 .}

1. $d$ não é simétrica. De fato, considere $O$ como sendo a origem e $P$ qualquer ponto em $\mathbb{B}^{2}$ distinto de $O$. Assim, observamos:

$$
d(O, P)=-\ln (1-\|P\|) \neq \ln (1+\|P\|)=d(P, O) .
$$

O Teorema 5.1, a seguir, fornece uma melhor visualização dessa anti-simetria.

2. $d$ não é invariante por translações. De fato, considere $T: \mathbb{B}^{2} \rightarrow \mathbb{B}^{2}$ dada por $T(x)=x+P_{0}$ onde $P_{0} \in B^{2} \backslash\{O\}$. Note-se

$$
d\left(O,-P_{0}\right)=d\left(O, P_{0}\right) \neq d\left(P_{0}, O\right)=d\left(T(O), T\left(-P_{0}\right)\right)
$$

3. dé invariante por rotações. Basta notar que o produto interno euclidiano e a norma euclidiana são invariantes por rotações. 


\section{Geometria da métrica de Funk sobre $\mathbb{B}^{2}$}

Nesta seção, examinaremos algumas propriedades geométricas da geometria básica, como a distância de um ponto a outro, distância de ponto a reta e o estudo da circunferência, utilizando a distância obtida em (29) induzida pela métrica de Funk (9).

A expressão (29) é um tanto complicada, porém ela pode ser reescrita de uma maneira bem mais tratável, conforme observaremos a continuação:

Teorema 5.1. Sejam $P, Q$ pontos de $\mathbb{B}^{2}$ distintos e $t_{2}$ definida por (19). Denotemos

$$
r=\frac{t_{2}}{t_{2}-1}>1
$$

então $P, Q$ e $r$ satisfazem a seguinte igualdade

$$
\left\|Q-\frac{P}{r}\right\|=\frac{r-1}{r} .
$$

Prova. Substituindo (16) em (19), temos:

$$
t_{2}=\frac{\sqrt{k}-\langle P, Q-P\rangle}{\|Q-P\|^{2}} .
$$

Substituindo (32) em 30, temos:

$$
r=\frac{\sqrt{k}-\langle P, Q-P\rangle}{\sqrt{k}-\langle P, Q-P\rangle-\|Q-P\|^{2}} .
$$

Logo, multiplicando $\sqrt{k}-\langle P, Q-P\rangle-\|Q-P\|^{2}$ ambos os lados de (33), tem-se:

$$
\begin{gathered}
r\left(\sqrt{k}-\langle P, Q-P\rangle-\|Q-P\|^{2}\right)=\sqrt{k}-\langle P, Q-P\rangle \\
(r-1)(\sqrt{k}-\langle P, Q-P\rangle)=r\|Q-P\|^{2} \\
\sqrt{k}=\langle P, Q-P\rangle+\left(\frac{r}{r-1}\right)\|Q-P\|^{2}
\end{gathered}
$$

evidenciando $k$ :

$$
\begin{aligned}
k & =\left(\langle P, Q-P\rangle+\left(\frac{r}{r-1}\right)\|Q-P\|^{2}\right)^{2} \\
& =\langle P, Q-P\rangle^{2}+\left(\frac{r}{r-1}\right)^{2}\|Q-P\|^{4}+2\left(\frac{r}{r-1}\right)\langle P, Q-P\rangle\|Q-P\|^{2}
\end{aligned}
$$

e comparando-a com 16, temos:

$$
\left(1-\|P\|^{2}\right)\|Q-P\|^{2}=\left(\frac{r}{r-1}\right)^{2}\|Q-P\|^{4}+2\left(\frac{r}{r-1}\right)\langle P, Q-P\rangle\|Q-P\|^{2}
$$


como $P \neq Q$ podemos simplificar $\|Q-P\|^{2}$ a ambos os lados da igualdade anterior:

$$
\begin{gathered}
1-\|P\|^{2}=\left(\frac{r}{r-1}\right)^{2}\|Q-P\|^{2}+2\left(\frac{r}{r-1}\right)\langle P, Q-P\rangle \\
1=\left(\frac{r}{r-1}\right)^{2}\|Q-P\|^{2}+2\left(\frac{r}{r-1}\right)\langle P, Q-P\rangle+\|P\|^{2} \\
\left(\frac{r-1}{r}\right)^{2}=\|Q-P\|^{2}+2\left(\frac{r-1}{r}\right)\langle P, Q-P\rangle+\left(\frac{r-1}{r}\right)^{2}\|P\|^{2} .
\end{gathered}
$$

Agora, completando quadrados:

$$
\begin{gathered}
\left(\frac{r-1}{r}\right)^{2}=\left\|(Q-P)+\left(\frac{r-1}{r}\right) P\right\|^{2} \\
\left(\frac{r-1}{r}\right)^{2}=\left\|Q-\frac{P}{r}\right\|^{2}
\end{gathered}
$$

Portanto, temos:

$$
\left\|Q-\frac{P}{r}\right\|=\frac{r-1}{r}
$$

Observa-se que a equação (31) poderia ser encontrada sem a hipótese de estarmos no plano $\mathbb{R}^{2}$, isto é, $P, Q$ poderiam ser pontos de $\mathbb{R}^{n}$ na bola unitária.

Observação 5.2. Veja que usando a equação (31) é mais fácil mostrar que $d$ é invariante por rotações, isto é,

$$
d(R P, R Q)=d(P, Q)
$$

onde $P, Q \in \mathbb{B}^{2}$ e $R=\left(\begin{array}{cc}\cos \theta & \operatorname{sen} \theta \\ -\operatorname{sen} \theta & \cos \theta\end{array}\right)$.

Observação 5.3. Consideremos $P=(0,0)$ em 31 , então

$$
\|Q\|=\frac{r-1}{r} .
$$

Quando $\|Q\| \rightarrow 1$, isto é, quando $Q$ se aproxima na borda de $\mathbb{B}^{2}$ então $r \rightarrow+\infty$, consequentemente $d(P, Q) \rightarrow+\infty$. Isso significa, junto à propriedade 2. na Observação 2.8, que a partir de qualquer ponto em $\mathbb{B}^{2}$, nosso barco não conseguirá sair de $\mathbb{B}^{2}$. Por outro lado, consideremos $Q=(0,0)$ em (31), então

$$
\|P\|=r-1
$$

Quando $\|P\| \rightarrow 1$, então $r \rightarrow 2$, consequentemente $d(P, Q) \rightarrow \ln (2)$. Logo, a partir da borda de $\mathbb{B}^{2}$, nosso barco consegue chegar até a origem em um tempo igual a ln 2. 


\subsection{Circunferência}

Sendo que a distância de Funk não é simétrica, isto é, $d_{F}(P, Q) \neq d_{F}(Q, P)$, temos duas interpretações para a noção de circunferência, estes são, a "saída" do centro, chamada de tipo 1; e a "entrada" ao centro, denominada de tipo 2.

Definição 5.4. Dados $P$ um ponto de $\mathbb{B}^{2}$ e $r \geq 1$ um número real, definimos a circunferência de Funk tipo 1 , de centro $P$ e raio $\ln r$, como sendo os pontos $Q \in \mathbb{B}^{2}$ tais que satisfazem a seguinte equação:

$$
d_{F}(P, Q)=\ln r
$$

Por (31), temos que a equação da circunferência tipo 1 de Funk de centro $P=(a, b)$ e raio $\ln r$, é dada por:

$$
\left(x_{1}-\frac{a}{r}\right)^{2}+\left(x_{2}-\frac{b}{r}\right)^{2}=\left(\frac{r-1}{r}\right)^{2} .
$$

Claramente a equação (35) descreve o gráfico de uma circunferência euclidiana em $\mathbb{R}^{2}$ com centro em $\frac{P}{r}$ e raio $\frac{r-1}{r}$. Em outras palavras, a circunferência euclidiana de centro em $P$ e raio $\ln r$ foi deslocada a uma taxa de $1-\frac{1}{r}$ em direção à origem e contraída a uma taxa de $1-\frac{r-1}{r \ln r}$.

Exemplo 5.5. Dados o centro $P=(0,0)$ e $r=e$. Obtemos de (35) a equação da circunferência $c$ tipo 1 de centro $P$ e raio 1.

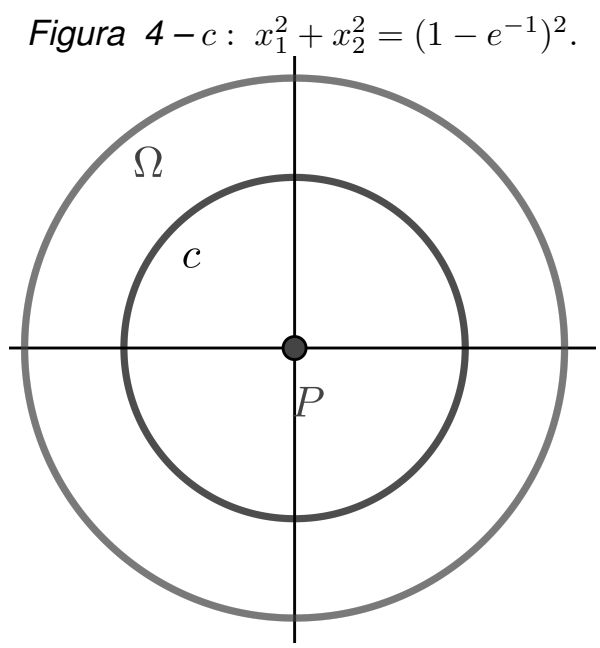

Fonte: Elaboração dos autores (2020).

Exemplo 5.6. Dados $P=\left(\frac{1}{2}, \frac{1}{2}\right)$ e $r=e$. Obtemos de (35) a equação da circunferência $c$ tipo 1 de centro $P$ e raio igual 1 . 


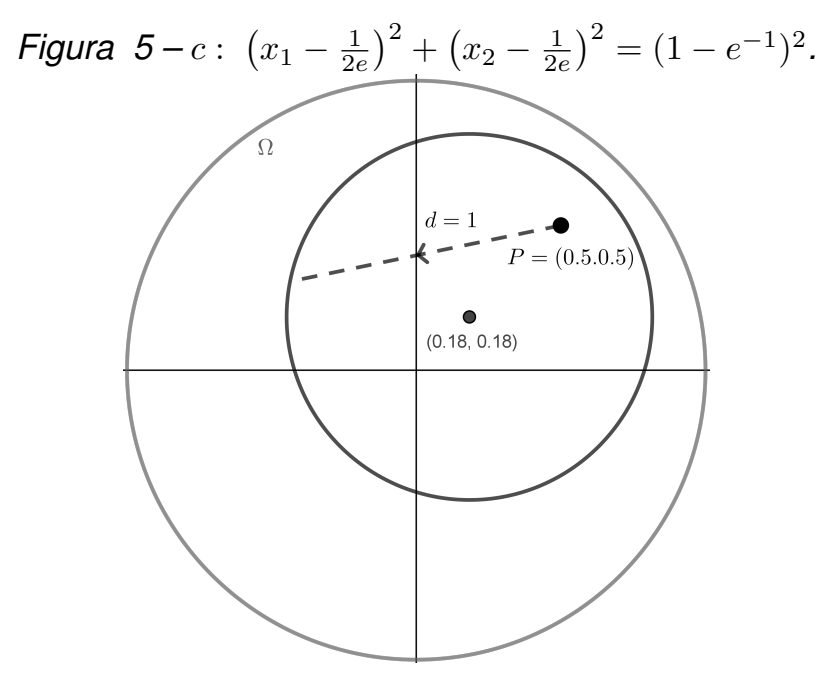

Fonte: Elaboração dos autores (2020).

Definição 5.7. Dados $P$ um ponto de $\mathbb{B}^{2} \subset \mathbb{R}^{2}$ e $r \geq 1$ um número real, definimos a circunferência de Funk tipo 2, de centro $P$ e raio $\ln r$, como sendo os pontos $Q \in \mathbb{B}^{2}$ tais que satisfazem a seguinte equação:

$$
d_{F}(Q, P)=\ln r
$$

Por (31), temos que a equação da circunferência tipo 2 de Funk de centro $P=(a, b)$ e raio $\ln r$, é dada por:

$$
\left(a-\frac{x_{1}}{r}\right)^{2}+\left(b-\frac{x_{2}}{r}\right)^{2}=\left(\frac{r-1}{r}\right)^{2}
$$

ou, equivalentemente,

$$
\left(x_{1}-r a\right)^{2}+\left(x_{2}-r b\right)^{2}=(r-1)^{2} .
$$

Note que (36) é uma circunferência euclidiana com centro em $(r a, r b)$ e raio $r-1$.

Exemplo 5.8. Dados $P=\left(\frac{1}{2}, \frac{1}{2}\right)$ e $r=e$. Obtemos de (36) a equação da circunferência $c$ tipo 2 com centro em $P$ e raio igual 1 . 


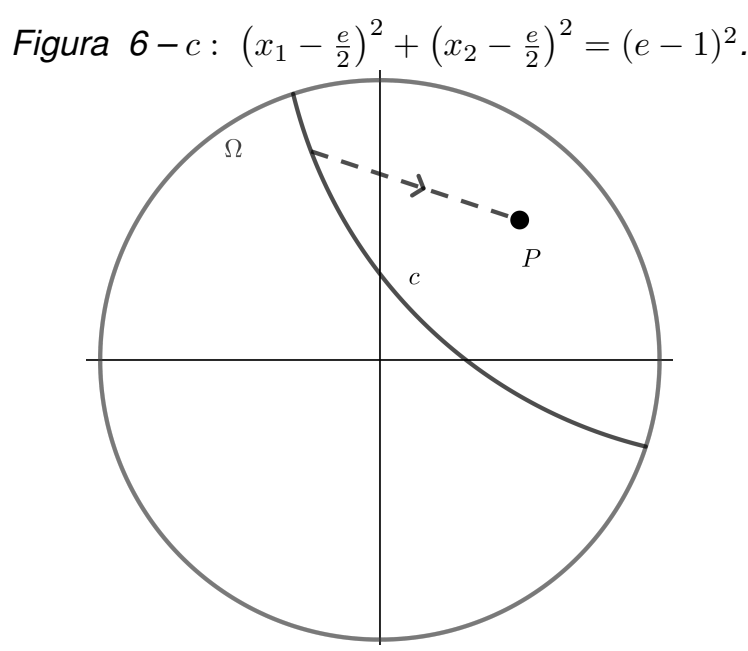

Fonte: Elaboração dos autores (2020).

\subsection{Distância de Funk de reta a ponto}

Definição 5.9. Definimos a distância, $d_{F}(s, Q)$, da reta $s$ até o ponto $Q$ por:

$$
d_{F}(s, Q):=\min \left\{d\left(P^{\prime}, Q\right) ; P^{\prime} \in s\right\} .
$$

Dizemos que o ponto $P * \in s$ realiza a distância de $s$ até o ponto $Q$, se

$$
d_{F}(P *, Q) \leq d_{F}\left(P^{\prime}, Q\right) \text {, para todo } P^{\prime} \in s .
$$

Dados uma reta $s: x_{2}=m x_{1}+c$ e um ponto $Q=(a, b) \in \mathbb{B}^{2}$, analisaremos o caso particular em que $m=0$ para depois analisar o caso geral. Para determinar a distância da reta $s$ ao ponto $Q$, tomamos uma circunferência de Funk, contendo $Q$ e com centro em algum ponto da reta. O raio é $\ln r$ e centro em $P=\left(x_{1}, c\right) \in s$. De acordo com a equação (36), temos:

$$
\left(x_{1}-r a\right)^{2}+(c-r b)^{2}=(r-1)^{2} .
$$

Essa é uma equação de segundo grau na variável $x_{1}$, e dependendo de $r$, é possível encontrar duas, uma ou nenhuma solução para $x_{1}$. Estamos interessados apenas em encontrar uma única solução, aquela que minimiza a distância. Assim, da equação (37) temos as condições de unicidade, $x_{1}=r a \mathrm{e}$

$$
(c-r b)^{2}=(r-1)^{2} .
$$

Desenvolvendo os quadrados obtemos uma equação de segundo grau em $r$ :

$$
\left(1-b^{2}\right) r^{2}+2(b c-1) r+\left(1-c^{2}\right)=0,
$$


cujas raízes estão dadas por:

$$
r=\frac{(1-b c) \pm \sqrt{(1-b c)^{2}-\left(1-b^{2}\right)\left(1-c^{2}\right)}}{\left(1-b^{2}\right)}
$$

Nota-se que:

$$
\begin{aligned}
(1-b c)^{2}-\left(1-b^{2}\right)\left(1-c^{2}\right) & =1-2 b c+b^{2} c^{2}-\left(1-c^{2}-b^{2}+b^{2} c^{2}\right) \\
& =c^{2}-2 b c+b^{2}=(c-b)^{2}
\end{aligned}
$$

Dessa forma, a equação 38 torna-se $r=\frac{1-b c \pm|c-b|}{1-b^{2}}$. Lembrando que $r>1$, obtemos:

$$
r=\frac{1-b c+|c-b|}{1-b^{2}}
$$

Assim, a distância de Funk da reta $s$ ao ponto $Q=(a, b)$, para o caso em que $m=0$, é dada por:

$$
d_{F}(s, Q)=\ln r=\ln \left(\frac{1-b c+|c-b|}{1-b^{2}}\right)
$$

Essa distância é realizada pelo ponto $P *=(a r, c) \in s$.

Veremos, adiante, como determinar essa distância para uma reta $s$ qualquer. Para isso, observamos primeramente, que o campo de forças é simétrico em relação a origem (ver a equação (34)). Assim, podemos aplicar apenas uma rotação nos eixos e encontrar valores para $x_{1}$ e $r$ em relação aos resultados já obtidos.

Dada uma reta $s: x_{2}=m x_{1}+c$, rotacionamos os eixos coordenados $\theta$ graus, tal que $m=\tan \theta$. Note que, nos novos eixos rotacionados, a reta $s$ é uma reta com inclinação nula (paralela ao eixo $x_{1}$ ), recaindo ao caso particular em que $m=0$. Determinaremos as novas coordenadas de $Q=(a, b)$ :

$$
\left(\begin{array}{l}
a^{\prime} \\
b^{\prime}
\end{array}\right)=\left(\begin{array}{cc}
\cos \theta & \operatorname{sen} \theta \\
-\operatorname{sen} \theta & \cos \theta
\end{array}\right)\left(\begin{array}{l}
a \\
b
\end{array}\right)
$$

Logo, as novas coordenadas de $Q$ são $(a \cos \theta+b \operatorname{sen} \theta, b \cos \theta-a \operatorname{sen} \theta)$.

De 37) e (39), obtemos:

$$
r=\frac{1-c \cos \theta(b \cos \theta-a \operatorname{sen} \theta)+|c \cos \theta-b \cos \theta+a \operatorname{sen} \theta|}{1-(b \cos \theta-a \operatorname{sen} \theta)^{2}} \text { e } x=r(a \cos \theta+b \operatorname{sen} \theta) .
$$


Assim, obtemos a distância de Funk da reta $s$ ao ponto $Q$, dada por:

$$
d_{F}(s, Q)=\ln r=\ln \left(\frac{1-c \cos \theta(b \cos \theta-a \operatorname{sen} \theta)+|c \cos \theta-b \cos \theta+a \operatorname{sen} \theta|}{1-(b \cos \theta-a \operatorname{sen} \theta)^{2}}\right) .
$$

Mais ainda, essa distância é realizada pelo ponto $P * *=(r(a \cos \theta+b \operatorname{sen} \theta), c \cos \theta)$.

Para obter o ponto sobre a reta original (sem a rotação) é suficiente rotar no sentido contrário:

$$
P *=\left(\begin{array}{cc}
\cos \theta & -\operatorname{sen} \theta \\
\operatorname{sen} \theta & \cos \theta
\end{array}\right)\left(\begin{array}{c}
a r \cos \theta+b r \operatorname{sen} \theta \\
c \cos \theta
\end{array}\right) .
$$

Exemplo 5.10. Dados o ponto $Q=\left(\frac{1}{2}, 0\right)$ e a reta $s: x_{2}=m x_{1}+c \operatorname{com} m=\tan \left(46^{\circ}\right)$ e $c=0.5$. De (40) e (41) temos $d(s, Q) \approx \ln (2.1)$ e $P * \approx(0.26,0.77)$, respectivamente.

Figura 7 - Distância Funk reta a ponto.

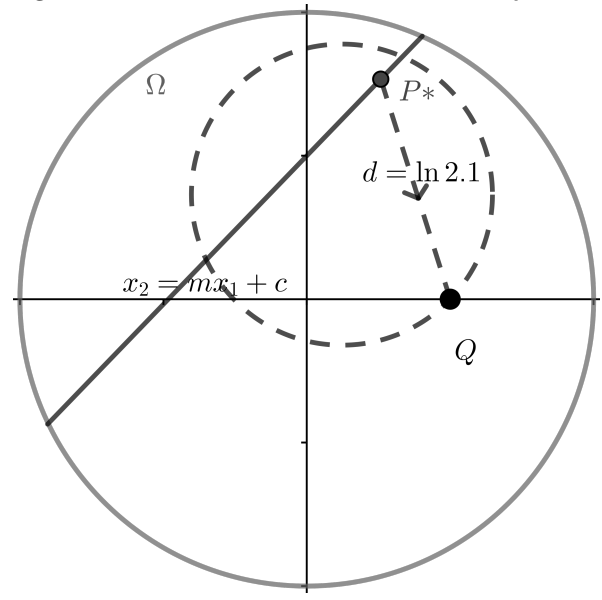

Fonte: Elaboração dos autores (2020).

\subsection{Distância de Funk de ponto a reta}

Definição 5.11. Definimos a distância, $d_{F}(P, s)$, do ponto $P$ até a reta $s$ por:

$$
d_{F}(P, s):=\min \left\{d\left(P, P^{\prime}\right) ; P^{\prime} \in s\right\} .
$$

Dizemos que o ponto $P * \in s$ realiza a distância de $P$ até a reta $s$, se

$$
d_{F}(P, P *) \leq d_{F}\left(P, P^{\prime}\right) \text {, para todo } P^{\prime} \in s .
$$

Dados o ponto $P=(a, b)$ e a reta $s: x_{2}=m x_{1}+c$, faremos de forma semelhante ao que fizemos anteriormente para determinar a distância de Funk de reta ao ponto. Tomamos uma 
circunferência de Funk com centro em $P$, raio $\ln r$ e com interseção com a reta $s$. Tomamos o ponto $Q \in s$ sendo uma dessas interseções. Para tanto, verificaremos, primeiramente, o caso particular em que $m=0$. Logo, pela equação (31), temos:

$$
\left(x_{1}-\frac{a}{r}\right)^{2}+\left(c-\frac{b}{r}\right)^{2}=\left(\frac{r-1}{r}\right)^{2} \text {. }
$$

Analogamente, essa é uma equação de segundo grau na variável $x_{1}$, e dependendo de $r$. Entretanto, estamos interessados apenas em encontrar uma única solução, aquela que minimiza a distância. Assim, da equação (42) temos:

$$
r^{2} x_{1}^{2}-2 a r x_{1}+a^{2}+(c r-b)^{2}-(r-1)^{2}=0
$$

Para obter uma única solução, o discriminante da equação, de segundo grau em $x_{1}$, acima deve ser igual a zero. Consequentemente, teremos uma equação de segundo grau em $r$ :

$$
r^{2}\left(c^{2}-1\right)+2 r(1-b c)+b^{2}-1=0
$$

cujas raízes são dadas por:

$$
r=\frac{(b c-1) \pm \sqrt{(1-b c)^{2}-\left(c^{2}-1\right)\left(b^{2}-1\right)}}{\left(c^{2}-1\right)} .
$$

Observa-se:

$$
\begin{aligned}
(1-b c)^{2}-\left(c^{2}-1\right)\left(b^{2}-1\right) & =1-2 b c+b^{2} c^{2}-b^{2} c^{2}+c^{2}+b^{2}-1 \\
& =c^{2}-2 b c+b^{2}=(c-b)^{2}
\end{aligned}
$$

Assim, $r=\frac{b c-1 \pm|c-b|}{c^{2}-1}$. Para garantir que $r>1$, temos $r=\frac{b c-1-|c-b|}{c^{2}-1}$ e $x_{1}=\frac{a}{r}$. Logo, a distância de Funk do ponto $P$ a reta $s$ (caso em que $m=0$ ) é dada por:

$$
d_{F}(P, s)=\ln r=\ln \left(\frac{b c-1 \pm|c-b|}{c^{2}-1}\right) .
$$

Essa distância é realizada pelo ponto $Q *=\left(\frac{a}{r}, c\right)$.

Para o caso que $s$ é qualquer, basta rotacionar os eixos, como já fizemos antes. Assim, a distância de Funk do ponto $P$ a reta $s$ é dada por:

$$
d_{F}(P, s)=\ln r=\ln \left(\frac{c \cos \theta(b \cos \theta-1-a \operatorname{sen} \theta)-|c \cos \theta-b \cos \theta+a \operatorname{sen} \theta|}{c^{2} \cos ^{2} \theta-1}\right),
$$

realizada pelo ponto $Q * *=\left(\frac{a \cos \theta+b \operatorname{sen} \theta}{r}, c \cos \theta\right)$. 
Para obter o ponto sobre a reta original (sem a rotação) é suficiente rotar no sentido contrário:

$$
Q *=\left(\begin{array}{cc}
\cos \theta & -\operatorname{sen} \theta \\
\operatorname{sen} \theta & \cos \theta
\end{array}\right)\left(\begin{array}{c}
\frac{a}{r} \cos \theta+\frac{b}{r} \operatorname{sen} \theta \\
c \cos \theta
\end{array}\right) .
$$

Exemplo 5.12. Dados o ponto $P=\left(\frac{1}{2}, \frac{1}{2}\right)$ e a reta $s: x_{2}=m x_{1}+c \operatorname{com} m=\tan \left(30^{\circ}\right)$ e $c=0.6$. De (43) e (44), temos $d(P, s) \approx \ln (1.67)$ e $Q * \approx(0.09,0.65)$, respectivamente.

Figura 8 -Distância Funk ponto a reta.

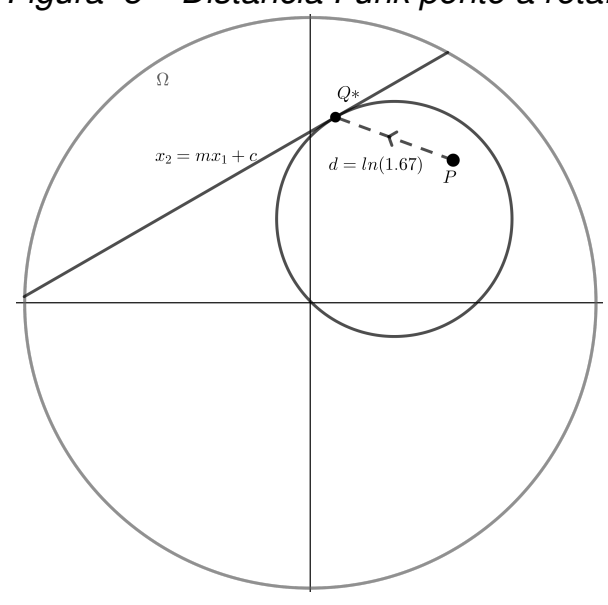

Fonte: Elaboração dos autores (2020).

\section{Considerações finais}

O problema de navegação de Zermelo estudado neste trabalho trata de um barco navegando sobre um disco unitário a uma velocidade constante considerando correntezas de vento na direção do centro, o equivalente para este problema de navegação recai no estudo da métrica de Funk sobre o disco unitário, que é uma métrica particular das métricas de Randers, que por sua vez são um caso particular das métricas de Finsler. Esta nova forma de ver o problema nos dá uma fórmula da distância de um ponto a outro um pouco complicada de lidar, mas uma equivalência mais tratável e mais intuitiva é dada no teorema principal. Com ajuda deste teorema conseguimos resolver os problemas propostos no início deste trabalho como são: equação da circunferência tipo 1 e tipo 2, distância de ponto a reta e de reta a ponto. Encorajamos ao leitor obter a distância de uma reta a outra.

Questões naturais surgem para continuidade deste trabalho. Por exemplo: Como são as equações das cônicas nesta métrica? É possível definir ângulo? 
As métricas de Finsler são uma área da geometria relativamente nova e Shiing-Shen Chern, chamado pai da geometria diferencial moderna, defendia a inclusão da geometria de Finsler na grade curricular das universidades. Este trabalho é uma pequena contribuição ao sonho de S. S. Chern.

\section{Referências}

CARMO, M. P. do. Geometria Diferencial de Curvas e Superfícies. 6. ed. Rio de Janeiro: SBM, 2012.

CARMO, M. P. do. Geometria Riemanniana. 5 ed. Rio de janeiro: SBM, 2011.

CHENG, X.; SHEN, Z. Finsler Geometry: An approach via Randers spaces. Beijing-Heidelberg: Science Press Beijing-Springer, 2012.

CHERN, S. S.; SHEN, Z. Riemannian-Finsler geometry. Singapore: World Scientific, 2005.

FUNK, P. Über Geometrien, bei denen die Geraden die Kürzesten sind. Mathematische Annalen, n. 101, p. 226-237, 1929.

LIMA, E. L. Análise Real. v. 1. Coleção Matemática Universitária. 12. ed. Rio de Janeiro: IMPA, 2014.

SHEN, Z. Lectures on Finsler Geometry. Singapore: World Scientific, 2001. 